\title{
On Reverse Super Edge Magic Total Labeling of Subdivided Trees
}

\author{
Abdul Raheem \\ Department of Mathematics, \\ National University of Singapore, Singapore 119076 \\ rahimciit7@gmail.com
}

\begin{abstract}
A reverse edge magic total labeling of a graph $G$ is a one-to-one map $\lambda: V(G) \cup E(G) \rightarrow$ $\{1,2, \ldots,|V(G) \cup E(G)|\}=[1,|V(G) \cup E(G)|]$ with the property that there is an integer constant $k$ such that $\{\lambda(x y)-(\lambda(x)+\lambda(y)) / x y \in V(G)\}=k$. If $(V(G))=[1,|V(G)|]$ then the reverse edge magic labeling is called reverse super edge-magic labeling. In this paper we will formulate the reverse edge magic labeling of two subclasses of trees.
\end{abstract}

Keywords: star, subdivision of star, reverse super edge magic labeling

\section{Introduction}

All graphs in this paper are finite, undirected, simple and planar. The graph $G$ has the vertex set $\mathrm{V}(\mathrm{G})$ and the edge set $\mathrm{E}(\mathrm{G})$. A (v,e) -graph $\mathrm{G}$ is a graph such that $|\mathrm{V}(\mathrm{G})|=\mathrm{v}$ and $|\mathrm{E}(\mathrm{G})|=\mathrm{e}$. A general reference for graph-theoretic ideas can be seen in [1,2]. A labeling (or valuation) of a graph is a map that converts graph elements into numbers (usually to positive or non-negative integers). In this paper, the domain will be the set of all vertices and edges and such a labeling is known as total labeling. Some labeling alternatively uses either the vertex-set or the edge-set and we shall address them as vertex-labeling or edge-labeling, respectively.

Definition 1.1. A reverse edge magic total labeling of a graph $G$ is a one-to-one map $\lambda: V(G) \cup$ $E(G) \rightarrow\{1,2, \ldots,|V(G) \cup E(G)|\}=[1,|V(G) \cup E(G)|]$ with the property that there is an integer constant $k$ such that $\{\lambda(x y)-(\lambda(x)+\lambda(y)) / x y \in V(G)\}=k$.

Definition 1.2. $\lambda$ is called the reverse super edge magic total labeling and $G$ is known as a reverse super edge magic total graph. Enomoto et al. [3] proposed the following conjecture,

Conjecture 1.1. Every tree admits a super edge magic total labeling.

In favor of this conjecture, many authors have considered a super edge magic total labeling for different classes of trees. For detailed studies the reader can see [4-14].

Definition 1.3. Let $n_{i} \geq 1,1 \leq i \leq r$ and $r \geq 2$. A subdivided $\operatorname{star} S b\left(n_{1}, n_{2}, \ldots, n_{r}\right)$ is a tree obtained by inserting $n_{i}-1$ nodes to each of the ith edge of the star $K_{1, r}$. Let us define the set of nodes and edges as follows,

$V(G)=\{c\} \cup\left\{x_{i}{ }^{l} \mid 1 \leq i \leq r ; 1 \leq l \leq n_{i}\right\}$ and $E(G)=\left\{c x_{i}{ }^{l} \mid 1 \leq i \leq r\right\} \cup\left\{x_{i}{ }^{l_{i}} x_{i}{ }^{l_{i}+1} \mid 1 \leq i \leq\right.$ $\left.r ; 1 \leq l \leq n_{i}-1\right\}$.

However, the investigation of different results related to a reverse super edge magic total labeling of the subdivided star $S b\left(n_{1}, n_{2}, \ldots, n_{r}\right)$ for $n_{1} \neq n_{2}, \ldots, \neq n_{r}$ is still an open problem. In this paper, we will formulate a reverse super edge magic total labeling of the subclasses of 
subdivided stars denoted by $S b\left(m n, m n, m n, 2 m n, n_{6}, n_{r}, \ldots, n_{r}\right)$ and $S b(m n, m n .2 n, 2 n+$ $\left.2,4 n+3, n_{6}, \ldots, n_{r}\right)$ under certain conditions.

Let us consider the following Lemma which we will use frequently in the main theorems.

Lemma 1.1. A graph with vertices $v$ and e edges is reverse super edge magic total labeling if and only if there exists a bijective function $\lambda: V(G) \rightarrow[1, v]$ such that the set consists of consecutive integers. In such a case, $\lambda$ extends to a reverse super edge magic total labeling of $\mathrm{G}$ with magic constant $k=2 v-s-1$, where $s=\max (S)$.

\section{Main Results}

In this section, we will prove the main results related to a reverse super edge magic total labeling of the more generalized subclasses of subdivided trees.

Theorem 2.1. The graph $G \cong S b\left(n, n, n, n, 2 n, n_{6}, \ldots, n_{r}\right)$ admits the reverse edge magic total labeling for any odd $n \geq 3, r \geq 6, n_{p}=2^{p-4} n-2 p+11$ and $6 \leq p \leq r$

Proof Let $v=|V(G)|$ and $e=|E(G)|$ then

$v=6 n+1+\sum_{t=6}^{r}\left[2^{t-4} n-2 t\right]$ and $e=v-1$

Let us define $\lambda: V(G) \rightarrow[1, v]$ as follows,

$$
\lambda(c)=(4 n+2)+\sum_{t=6}^{r}\left[2^{t-5} n-t+6\right]
$$

For odd $1 \leq l \leq n_{i}$, where $1 \leq i \leq 5$ and for $1 \leq i \leq r$ :

$$
\lambda(w)=\left\{\begin{array}{cc}
\frac{l_{1}+1}{2}, \quad \text { if } w=x_{1} l_{1} \\
n+1-\frac{l_{2}-1}{2}, \quad \text { if } w=x_{2}{ }^{l_{2}} \\
(n+2)+\frac{l_{3}-1}{2}, & \text { if } w=x_{3}{ }^{l_{3}} \\
2(n+1)+\frac{l_{4}-1}{2}, & \text { if } w=x_{4}{ }^{l_{4}} \\
(3 n+2)-\frac{l_{5}-1}{2}, & \text { if } w=x_{5}{ }^{l_{5}}
\end{array}\right.
$$

$\lambda\left(x_{i}{ }^{l_{i}}\right)=(3 n+2)+\sum_{t=6}^{i}\left[2^{t-5} n-t+6\right]-\frac{l_{i}-1}{2}$ Accordingly.

For even $1 \leq l \leq n_{i}$ and $\gamma=(3 n+2)+\sum_{t=6}^{r}\left[2^{t-6} n+1\right]$, where $1 \leq i \leq 5$ and for $6 \leq i \leq$ $r$ : 


$$
\lambda(w)=\left\{\begin{array}{cc}
(\gamma+1) \frac{l_{1}-2}{2}, \quad \text { if } w=x_{1}{ }^{l_{1}} \\
(\gamma+n-1)-\frac{l_{2}-2}{2}, & \text { if } w=x_{2}{ }^{l_{2}} \\
(\gamma+n+1)+\frac{l_{3}-2}{2}, & \text { if } w=x_{3}{ }^{l_{3}} \\
(\gamma+2 n-1)+\frac{l_{4}-2}{2}, & \text { if } w=x_{4}{ }^{l_{4}} \\
(\gamma+3 n-1)-\frac{l_{5}-2}{2}, & \text { if } w=x_{5}{ }^{l_{5}}
\end{array}\right.
$$

$\lambda\left(x_{i}^{l_{i}}\right)=(\gamma+3 n-1)+\sum_{t=6}^{i}\left[2^{t-5}(3 n)-2 t+11\right]-\frac{l_{i}-2}{2}$ Accordingly.

By using the above scheme of labeling, we get the set of edge-sums consecutive integer sequence $S=[\gamma+2, \gamma+1+e]$. Therefore, by Lemma 1.1 can be extended to a reverse edge magic total labeling with magic constant $k=(3 n-2)+\sum_{t=6}^{i}\left[2^{t-6}(3 n)-2 t+10\right]$.

Theorem 2.2. The graph $G \cong T\left(3 n, 3 n, 3 n, 3 n, 6 n, n_{6}, \ldots, n_{r}\right)$ admits the reverse edge magic total labeling with $k=2 v-s-1$ for any odd $n \geq 3, r \geq 6, n_{p}=2^{t-6}(3 n)-2 t+11$ and $6 \leq$ $p \leq r$

\section{Proof}

Let Let $v=|V(G)|$ and $e=|E(G)|$ then

$v=(18 n+1)+\sum_{t=6}^{r}\left[2^{t-4}(3 n)-2 t+11\right]$ and $e=v-1$

Let us define $\lambda: V(G) \rightarrow[1, v]$ as follows,

$$
\lambda(c)=(12 n+2)+\sum_{t=6}^{r}\left[2^{t-5}(3 n)-t+6\right]
$$

For odd $1 \leq l \leq n_{i}$, where $1 \leq i \leq 5$ and for $1 \leq i \leq r$ :

$$
\lambda(w)=\left\{\begin{array}{cc}
\frac{l_{1}+1}{2}, \quad \text { if } w=x_{1}{ }^{l_{1}} \\
3 n+1-\frac{l_{2}-1}{2}, \quad \text { if } w=x_{2}{ }^{l_{2}} \\
(3 n+2)+\frac{l_{3}-1}{2}, & \text { if } w=x_{3}{ }^{l_{3}} \\
(6 n+2)-\frac{l_{4}-1}{2}, & \text { if } w=x_{4}{ }^{l_{4}} \\
(9 n+2)-\frac{l_{5}-1}{2}, & \text { if } w=x_{5}{ }^{l_{5}}
\end{array}\right.
$$

$\lambda\left(x_{i} l_{i}\right)=(9 n+2)+\sum_{t=6}^{i}\left[2^{t-5}(3 n)-t+6\right]-\frac{l_{i}-1}{2}$ Accordingly.

For even $1 \leq l \leq n_{i}$ and $\gamma=(9 n+2)+\sum_{t=6}^{r} 2^{t-5}(3 n)-t+6$, where $1 \leq i \leq 5$ and for $6 \leq$ $i \leq r$ : 


$$
\lambda(w)=\left\{\begin{array}{cc}
(\gamma+1) \frac{l_{1}-2}{2}, \quad \text { if } w=x_{1}{ }^{l_{1}} \\
(\gamma+3 n-1)-\frac{l_{2}-2}{2}, & \text { if } w=x_{2}{ }^{l_{2}} \\
(\gamma+3 n+1)+\frac{l_{3}-2}{2}, & \text { if } w=x_{3}{ }^{l_{3}} \\
(\gamma+6 n-1)-\frac{l_{4}-2}{2}, & \text { if } w=x_{4}{ }^{l_{4}} \\
(\gamma+9 n-1)-\frac{l_{5}-2}{2}, & \text { if } w=x_{5}{ }^{l_{5}}
\end{array}\right.
$$

$\lambda\left(x_{i}^{l_{i}}\right)=(\gamma+9 n-1)+\sum_{t=6}^{i}\left[2^{t-5}(3 n)-2 t+11\right]-\frac{l_{i}-2}{2}$ Accordingly

By using the above scheme of labeling, we get the set of edge-sums consecutive integer sequence $S=[\gamma+2, \gamma+1+e]$. Therefore, by Lemma 1.1 can be extended to a reverse edge magic total labeling with magic constant $k=(9 n-2)+\sum_{t=6}^{i}\left[2^{t-6}(3 n)-t+5\right]$.

Theorem 2.3 The graph $G \cong S b\left(m n, m n, m n, 2 m n, n_{6}, \ldots, n_{r}\right)$ admits the reverse edge magic total labeling with $k=2 v-s-1$ for any odd $n \geq 3, r \geq 6, n_{p}=2^{p-4} k n-2 p+11$ and $6 \leq$ $p \leq r$

\section{Proof}

Let Let $v=|V(G)|$ and $e=|E(G)|$ then

$v=(6 k n+1)+\sum_{t=6}^{r}\left[2^{m-4} k n-2 m+11\right]$ and $e=v-1$

Let us define $\lambda: V(G) \rightarrow[1, v]$ as follows,

$$
\lambda(c)=(4 k n+2)+\sum_{t=6}^{r}\left[2^{m-5} k n-m+6\right]
$$

For odd $1 \leq l \leq n_{i}$, where $1 \leq i \leq 5$ and for $1 \leq i \leq r$ :

$$
\lambda(w)=\left\{\begin{array}{cc}
\frac{l_{1}+1}{2}, \quad \text { if } w=x_{1}{ }^{l_{1}} \\
m n+1-\frac{l_{2}-1}{2}, \quad \text { if } w=x_{2}{ }^{l_{2}} \\
(m n+2)+\frac{l_{3}-1}{2}, \quad \text { if } w=x_{3}{ }^{l_{3}} \\
2(m n+2)-\frac{l_{4}-1}{2}, \quad \text { if } w=x_{4}{ }^{l_{4}} \\
(3 m n+2)-\frac{l_{5}-1}{2}, \quad \text { if } w=x_{5}{ }^{l_{5}}
\end{array}\right.
$$


For even $1 \leq l \leq n_{i}$ and $\gamma=(3 k n+2)+\sum_{t=6}^{r}\left[2^{t-6} 2 k n-(m-6)\right]$, where $1 \leq i \leq 5$ and for $6 \leq i \leq r$ :

$$
\lambda(w)=\left\{\begin{array}{cc}
(\gamma+1) \frac{l_{1}-2}{2}, \quad \text { if } w=x_{1}{ }^{l_{1}} \\
(\gamma+m n-1)-\frac{l_{2}-2}{2}, & \text { if } w=x_{2}{ }^{l_{2}} \\
(\gamma+m n+1)+\frac{l_{3}-2}{2}, & \text { if } w=x_{3}{ }^{l_{3}} \\
(\gamma+2 m n-1)-\frac{l_{4}-2}{2}, & \text { if } w=x_{4}{ }^{l_{4}} \\
(\gamma+3 m n-1)-\frac{l_{5}-2}{2}, & \text { if } w=x_{5}{ }^{l_{5}}
\end{array}\right.
$$

$\lambda\left(x_{i}{ }^{l_{i}}\right)=(\gamma+3 m n-1)+\sum_{t=6}^{i}\left[2^{t-5}(m n)-2 m+11\right]-\frac{l_{i}-2}{2}$ Accordingly.

By using the above scheme of labeling, we get the set of edge-sums consecutive integer sequence $S=[\gamma+2, \gamma+1+e]$. Therefore, by Lemma 1.1 can be extended to a reverse edge magic total labeling with magic constant $k=(3 m n-2)+\sum_{t=6}^{i}\left[2^{t-6}(m n)-t+10\right]$.

Theorem 2.4 The graph $G \cong S b\left(m n, m n .2 n, 2 n+2,4 n+3, n_{6}, \ldots, n_{r}\right)$ admits the reverse edge magic total labeling with $k=2 v-s-1$, for any odd $n \geq 3, r \geq 6, n_{p}=2^{p-5}(4 n+2)+1$ and $6 \leq p \leq r$

\section{Proof}

Let Let $v=|V(G)|$ and $e=|E(G)|$ then

$v=[(2 k+8) n+6]+\sum_{t=6}^{r}\left[2^{m-5}(4 n+2)+1\right]$ and $e=v-1$

Let us define $\lambda: V(G) \rightarrow[1, v]$ as follows,

$$
\lambda(c)=[(2 k+4) n+4]+\sum_{t=6}^{r}\left[2^{m-6}(4 n+2)+1\right]
$$

For odd $1 \leq l \leq n_{i}$, where $1 \leq i \leq 5$ and for $6 \leq i \leq r$ :

$$
\lambda(w)=\left\{\begin{array}{cc}
\frac{l_{1}+1}{2}, \quad \text { if } w=x_{1}{ }^{l_{1}} \\
m n+1-\frac{l_{2}-1}{2}, \quad \text { if } w=x_{2}{ }^{l_{2}} \\
(m n+2)+\frac{l_{3}-1}{2}, \quad \text { if } w=x_{3}{ }^{l_{3}} \\
(m+2) n+2-\frac{l_{4}-1}{2}, & \text { if } w=x_{4}{ }^{l_{4}} \\
(m+2) n+4-\frac{l_{5}-1}{2}, & \text { if } w=x_{5}{ }^{l_{5}}
\end{array}\right.
$$


$\lambda\left(x_{i}^{l_{i}}\right)=[(2 k+4) n+4]+\sum_{t=6}^{r}\left[2^{m-6}(4 n+2)+1\right] \frac{l_{i}-1}{2}$ Accordingly.

For even $1 \leq l \leq n_{i}$ and $\gamma=(3 k n+2)+\sum_{t=6}^{r}\left[2^{t-6} 2 k n-(m-6)\right]$, where $1 \leq i \leq 5$ and for $6 \leq i \leq r$ :

$$
\lambda(w)=\left\{\begin{array}{c}
(\gamma+1) \frac{l_{1}-2}{2}, \quad \text { if } w=x_{1}{ }^{l_{1}} \\
(\gamma+m n-1)-\frac{l_{2}-2}{2}, \quad \text { if } w=x_{2}{ }^{l_{2}} \\
(\gamma+m n+1)+\frac{l_{3}-2}{2}, \quad \text { if } w=x_{3}{ }^{l_{3}} \\
(\gamma+(m+2) n-1)-\frac{l_{4}-2}{2}, \quad \text { if } w=x_{4}{ }^{l_{4}} \\
(\gamma+(m+2) n+2)-\frac{l_{5}-2}{2}, \quad \text { if } w=x_{5}{ }^{l_{5}}
\end{array}\right.
$$

$\lambda\left(x_{i}^{l_{i}}\right)=[\gamma+(m+4) n+2]+\sum_{t=6}^{i}\left[2^{t-6}(4 n+2)+1\right]-\frac{l_{i}-2}{2}$ Accordingly.

By using the above scheme of labeling, we get the set of edge-sums consecutive integer sequence $S=[\gamma+2, \gamma+1+e]$. Therefore, by Lemma 1.1 can be extended to a reverse edge magic total labeling with magic constant $k=(m n+4)+\sum_{t=6}^{i}\left[2^{t-6}(4 n+2)\right]$.

\section{Conclusion}

In this paper, we have proved that the following subclasses of subdivided stars admit reverse super edge magic total labeling,

- For $n \geq 3$ and $k \geq 1$ are odd, $r \geq 6, S b\left(m n, m n, m n, 2 m n, n_{6}, \ldots, n_{r}\right)$ with $n_{p}=$ $2^{p-4}-2 p+11$ for $6 \leq p \leq r$.

- For $n \geq 3$ and $k \geq 1$ are odd, $r \geq 6, S b\left(m n, m n, 2 n, 2 n+2,4 n+3, n_{6}, \ldots, n_{r}\right)$ with $n_{p}=2^{p-5}(4 n+2)+1$ for $6 \leq p \leq r$.

The problem is still open for the remaining subclasses of subdivided stars with different combinations of $m$ and $n$.

\section{References}

[01] West DB. An introduction to graph theory. New Jersey: Prentice-Hall, 1996.

[02] Gallian JA. A dynamic survey of graph labeling. Electron J Combin. 2017;17:42-45.

[03] Enomoto H, Lladó, AS, Nakamigawa T, Ringel G. Super edge-magic graphs, SUT J Math. 1998;34:105-109.

[04] Baskoro ET, Sudarsana, IW, Cholily YM, How to construct new super edge-magic graphs from some old ones. J Indones Math Soc (MIHIM). 2005;11(2):155-162.

[05] Figueroa-Centeno RM, Ichishima R, Muntaner-Batle FA. On super edge-magic graph. Ars Combinatori. 2002;64: 81-95.

[06] Javaid M, Hussain M, Ali K, Dar KH. Super edge-magic total labeling on w-trees. Utilitas Math. 2011;86:183-191. 
[07] Ngurah AAG, Simanjuntak R, Baskoro ET. On (super) edge-magic total labeling of subdivision of $K_{1,3}$. SUT J Math. 2007;43:127-136.

[08] Salman ANM, Ngurah, AAG, Izzati N. On super edge-magic total labeling of a subdivision of a Star $S_{n}$. Utilitas Mthematica. 2010;81:275-284.

[09] Sharief BS. Reverse super edge magic labeling on w-trees. Int J Comput Eng Res Trends. 2015;2(11):719-721.

[10] Sharief BS. Madhusudhan RK. Reverse magic strength of festoon trees. Ital J Pure Appl Math. 2014;33:191-200.

[11] Raheem AM, Javaid M, Baig AQ. On antimagicness of subdivided stars. Discuss Math Graph Theory. 2015;35:663-673.

[12] Raheem A, Baig AQ. Antimagic labeling of the union of subdivided stars, TWMS J Appl Eng Math. 2016;6:244-250.

[13] Raheem A, Baig AQ, Javaid M. On (a,d)-EAT labeling of subdivision of $K_{1, r}$. J Inf Optim Sci. 2018;39(3):643-650.

[14] Venkata RS, Sharief BS. On reverse super edge-magic labeling of graphs, Int Rev Pure Appl Math. 2010;6(1):181-188. 